\title{
Stability under unanimous consent, free mobility and core*
}

\author{
Anna Bogomolnaia $^{\dagger} \quad$ Michel Le Breton ${ }^{\ddagger} \quad$ Alexei Savvateev $^{\S} \quad$ Shlomo Weber ${ }^{\llbracket}$
}

August 28, 2006

\begin{abstract}
In this paper we consider a population represented by a continuum of individuals uniformly distributed over the unit interval that faces a problem of location and financing of public facilities under the equal share rule. We examine three notions of stability of emerging jurisdiction structures (stability under unanimous consent, free mobility and core) and provide a characterization of stable structures.
\end{abstract}

Keywords: Jurisdiction structures, admission under unanimous consent, equal share, core, free mobility.

JEL Classification Numbers: D70, H20, H73.

\footnotetext{
${ }^{*}$ We are grateful to John Duggan and an anonymous referee for their useful comments and suggestions.

${ }^{\dagger}$ Rice University, Houston, USA.

${ }^{\ddagger}$ Université de Toulouse I, GREMAQ and IDEI, Toulouse, France.

${ }^{\S}$ Central Economics and Mathematics Institute, Moscow; Institute for Theoretical and Experimental Physics, Moscow; New Economic School, Moscow. Financial support through grants R98-0631 from the Economic Education and Research Consortium, \# NSh-1939.2003.6 School Support, Russian Foundation for Basic Research No. 04-0217227, and the Fund for Promotion of Russian Sciences is gratefully acknowledged.

"Southern Methodist University, Dallas, USA, and CORE, Catholic University of Louvain, Louvain-la-Neuve, Belgium.
} 


\section{Introduction}

Consider a population represented by a continuum of individuals uniformly distributed over the real line that faces a problem of location and financing of public facilities under its jurisdiction. More specifically, a decision is to be made on the total number of facilities to be built, where to locate them, how to assign each individual to a facility, and, finally, how to split the burden of financing of the facilities among the residents. Every resident faces two types of costs: one is an idiosyncratic transportation cost from their location to the chosen facility, and another is a monetary contribution to the costs of the facility she uses.

Thus, the group solution for the locational problem described here consists of jurisdiction structure, which is a partition of individuals into jurisdictions that consist of individuals assigned to the same facility; facility location in each jurisdiction, and sharing rule that determines individual contributions to cover the total cost of facilities in all jurisdictions.

In this paper we focus on the search for a stable partition of the entire population into several jurisdictions. ${ }^{1}$ In doing so, we impose the principles of efficiency and equal share. The efficiency requires that a location of the facility in each jurisdiction is chosen in order to minimize the total transportation cost of its residents. Since we assume that the transportation cost of each individual is proportional to her distance from the facility location, the efficiency requirement is equivalent to the majority voting requirement, and each jurisdiction places the facility at the location of its median resident. As in Cremer et al. (1985), Jéhiel and Scotchmer (1997, 2001), Alesina and Spolaore (1997), Casella (2001), Dahm et al. (2001), Haimanko et al. (2005), Bogomolnaia et al. (2005b), we impose the assumption of equal share, where all members of the same jurisdiction make equal contributions towards the facility cost. ${ }^{2}$

We then introduce several notions of stability that are immune to a possibility of groups of individuals migrating to one of the existing jurisdictions or creating a new one. It is important

\footnotetext{
${ }^{1}$ Throughout the paper, we will use the terms jurisdiction structure and partition interchangeably.

${ }^{2}$ See Haeringer (2000), Le Breton and Weber (2003), Haimanko et al. (2004), Le Breton et al. (2004), Bogomolnaia et al. (2005a) for alternative approaches to cost sharing mechanisms, and Le Breton and Weber (2004) for a general review of cost sharing schemes in this context.
} 
to stress that, while migrating between jurisdictions, all individuals anticipate the median location of the public facility and the equal share cost mechanism in a newly created jurisdiction. The most stringent stability notion we consider is what Jéhiel and Scotchmer (2001) call admission under unanimous consent (SAUC). This notion grants every individual the veto power regarding a possible migration of any group of individuals from other jurisdiction to her own. Obviously, the admission under unanimous consent severely restricts threats to stability, thus, generating a large set of stable jurisdiction structures. We then turn to the examination of more permissive stability threats. One is core stability (CS) or C-stable solution in Guesnerie and Oddou (1979), where every group of individuals is allowed to leave their jurisdictions and to create a new one. Another is stability under free mobility (SFM) that does not allow the members of an existing jurisdiction $S$ to prevent migration by members of other jurisdictions to $S$. While it is obvious that both CS and SFM notions are stronger than SAUC, we also examine a less obvious link between CS and SFM structures and show "almost every" SFM is also core stable. Even though there is one case, namely, of jurisdictions represented by intervals of the same length, where this relation is reverse, one can claim that in most of the cases the free mobility is the most permissive threat to stability.

An examination of stable partitions has to deal with the questions of number, size and composition of jurisdictions they contain. In characterizing the number of jurisdictions in a stable partition one has to take into account the conflict between increasing returns to scale that favor the creation of larger groups and the heterogeneity of individuals' locations that support the emergence of smaller groups. To determine the size and the composition of jurisdictions in a stable partition, one has to take into account the fact that the presence of a sufficient number of distant individuals in the jurisdiction may adversely impact the value of a total jurisdictional transportation cost to the chosen facility location. From this point of view, locational heterogeneity could be costly. Note that, for a given size of jurisdiction, the intra-heterogeneity is minimal when a jurisdiction is an interval. In local public finance, this feature is called stratification, while in game theory it is often called consecutiveness. Another important property of jurisdiction structures is inter-heterogeneity of jurisdiction sizes, or so-called heterogeneity gap in sizes of jurisdictions in stable partitions. We 
show that, unlike in Alesina and Spolaore (1997) and Casella (2001), our stability notions may yield a stable partition with sharply distinct jurisdiction sizes. Obviously, in the case of non-uniform distributions (see e.g, Bogomolanaia et al. (2005b) in the finite set-up) the heterogeneity of jurisdiction sizes is a natural feature of the model. What we show is that even the uniform distribution of individuals' locations may yield stable structures with sharply distinct jurisdiction sizes. ${ }^{3}$

Related literature. As we indicated above, some papers in this line of research examined various stability notions under the equal cost sharing rule and the uniform distribution of individuals over a bounded interval (Cremer et al. (1985), Jéhiel and Scotchmer (1997, 2001), Alesina and Spolaore (1997), Bogomolnaia et al. (2005b, 2006). Casella (2001) considered the model where individuals are distributed along the circle, whereas Dahm et al. (2001) studied a discrete model with equal distances between adjacent individuals. Haeringer (2000) and Le Breton and Weber (2003, 2004), Haimanko et al. (2004) considered a more general class of cost sharing schemes. A special attention has been paid to stability analysis under the Rawlsian principle of the utility maximization of the most disadvantaged individual (Haeringer (2000), Le Breton at al. (2004), Bogomolnaia et al. (2005a), Drèze et al. (2006). Haeringer (2001), Le Breton at al. (2003, 2004), Haimanko et al. (2004, 2005) have studied the environments with a more general class of distribution functions and addressed the question of characterization of cost sharing rules that admit a stable structure under various equilibrium notions.

The paper is organized as follows. Section 2 describes the model and introduces the notions of stability examined in this paper. In Section 3 we identify special jurisdiction structures that satisfy consecutiveness, border indifference, size monotonicity and homogeneity, and examine whether those properties are consistent with the stability notions introduced in previous section. In particular, we show that all SFM partitions are consecutive, whereas it is not necessarily the case for SAUC and CS partitions. Section 4 contains complete characterization results for SFM partitions and consecutive SAUC and CS partitions. More specifically, we establish bounds on the size of

\footnotetext{
${ }^{3}$ The heterogeneity of jurisdictional sizes has been examined by Jéhiel and Scotchmer $(1997,2001)$ who consider a setting where a public good ir represented by a single vertical dimension (quantity), and individuals are characterized by their willingness to pay for the public good.
} 
jurisdictions that yield stable partitions (under either SAUC, SFM or CS). It turns out that stable structures could display a strong size heterogeneity among jurisdictions that form it. We also establish a somewhat surprising link between SFM and CS (while both are weaker than SAUC). Namely, every SFM partition that contains jurisdictions of different sizes is also CS. However, the situation is reverse for partitions that consist of equal-size intervals, where every CS partition is SFM. Section 5 contains brief concluding remarks. The proofs of all results are relegated to the Appendix B. This is preceded by Appendix A that contains preliminary results and remarks.

\section{The Model}

We consider a society which faces the problem of location, financing and assignment of its members to public facilities (hospitals, schools, libraries, etc.). For that purpose, society may remain as a whole or be partitioned into several jurisdictions. Each jurisdiction selects the location for the facility (not necessarily within jurisdiction's bounds) and finances the cost of this facility by collecting tax from the jurisdiction members. Each individual therefore incurs two costs: the tax (her monetary contribution towards the costs of local facility), and the transportation cost from the individual's own location to that of the facility. The benefit of using the service is assumed to exceed any potential cost, and no individual would reject the usage of public facilities. This ensures the voluntarily participation of all individuals in the process described above.

We assume that the society consists of individuals uniformly distributed over the interval $I=$ $[0,1]$. Any measurable set $S \subset I$ of a positive Lebesgue measure (not necessarily an interval!) could be an admissible jurisdiction. We denote the measure of a jurisdiction $S$ by $|S|$. We call jurisdiction structure a partition $P=\left\{S_{i}\right\}_{1 \leq i \leq n}$ of $I$ into a finite number of jurisdictions. We refer to $P$ with $n$ elements as an $n$-partition. Slightly abusing the notation, we identify an individual with her location, so that we will use just $t$ for an individual located at the point $t \in I$.

The cost of a facility $g>0$ is independent of location and jurisdiction and is divided equally among jurisdiction members. The residents cover the cost of the facility so that the tax imposed on every member of the jurisdiction $S$ is $\frac{g}{|S|}$. Residents of each jurisdiction face an idiosyncratic 
cost proportional to the distance to the facility chosen by that jurisdiction. We assume that the transportation cost is linear in distance: an individual $t \in S$ faces the transportation cost $|t-s|$, if the facility is located at point $s$.

The efficiency condition implies that every jurisdiction $S$ locates the facility at its median point $m(S)$, which minimizes the total transportation cost of its members (see Haimanko et al. (2004)). If a median point is not unique (which can happen when $S$ is not a connected set), then there is an interval of median points and we assume that $m(S)$ is the midpoint of this interval.

Given the assumptions above, the total cost $c(t, S)$ of an individual $t$ in jurisdiction $S$ is uniquely given by:

$$
c(t, S)=|t-m(S)|+\frac{g}{|S|} .
$$

For any jurisdiction structure $P=\left\{S_{i}\right\}_{i=1}^{n}$ and any individual $t \in I$ we denote by $S^{t}$ the (unique) jurisdiction from $P$ which contains $t$. We will use the notation $c(t, P)$ for $c\left(t, S^{t}\right)$, the total cost an individual $t$ incurs in the jurisdiction structure $P$.

An arbitrarily chosen partition of the society could be prone to migrations by some dissatisfied groups (measurable sets) of individuals, who, in search for a better payoff (lower total cost) will switch to another jurisdiction, or even to form a new jurisdiction, Our goal is to identify stable partitions, immune to such migrations. We consider various notions of stability, steaming from three different principles for permissible group deviations (or migrations).

The first notion allows a group of individuals $S$ to join an existing jurisdiction $T$ whenever all migrants and all members of the migration target $T$ would benefit from the migration move. This notion of stability is called stability under admission by unanimous consent (Jéhiel and Scotchmer (2001)):

Definition 2.1: A partition $P$ is stable under admission by unanimous consent (SAUC) if there exists no group $S \subset I$ and a jurisdiction $T \in P$, such that $c(t, S \cup T)<c(t, P)$ for all $t \in S \cup T$.

In particular, this implies that no set of measure zero $S$ is allowed to move under SAUC condition. Indeed, in this case a migration move does impact the the members of $T$, thus violating the strict 
inequality in the definition. Thus, only a group of a positive measure could present a migration threat under SAUC.

The second notion of stability emerges when a group of individuals (from possibly different jurisdictions) is allowed to form a new jurisdiction. This leads to the traditional core stability (CS) notion which was called $C$-stable solution in Guesnerie and Oddou (1979):

Definition 2.2: A partition $P$ is called core stable $(C S)$ if there exists no group $S \subset I$ such that $c(t, S)<c(t, P)$ for every $t \in S$.

Since an admissible under SAUC migration is also an admissible deviation under CS, SAUC is a weaker requirement then CS.

The third possibility is to allow a group of individuals $S$ to join a jurisdiction $T$. It requires that only members of $S$ are better off, without demanding an improvement for members of $T$. The corresponding notion of stability is called stability under free mobility (SFM).

Definition 2.3: A partition $P$ is stable under free mobility (SFM) if there exists no group $S \in I$, together with a jurisdiction $T \in P$, such that $c(t, S \cup T)<c(t, P)$ for all $t \in S$.

Since SFM does not demand the consent of the members of the host jurisdiction, SAUC is weaker than SFM as well. The relation between SFM and CS is less obvious. ${ }^{4}$ Contrary to the CS requirement, under SFM, potential migrants have to join an existing jurisdiction and they are not allowed to form a new one. Nevertheless we will show that, except for the special case of partitions that consist of equal intervals, CS is implied by SFM. Thus, CS requirement is "generically" weaker then SFM. Even more surprisingly, in the homogenous case, the situation reverses and SFM is implied by CS.

To summarize the type of admissible deviations, under all three stability notions, a necessary condition for a group $S$ to consider a migration is the strict reduction of the after-migration relative to the pre-migration costs for all members of $S$. Moreover,

\footnotetext{
${ }^{4}$ Note that, unlike in SAUC, both CS and SFM allow for migrant sets of measure zero.
} 
- Under SAUC, a deviating group $S$ should join an existing jurisdiction $T$ and make all members of $T$ better off;

- Under CS, a deviating group $S$ should form its own new jurisdiction;

- Under SFM, a deviating group $S$ should join an existing jurisdiction, but there is no requirement of a cost reduction for the members of the host jurisdiction.

We now turn to identification of special classes of jurisdiction partitions and their compatibility with stability notions defined in this section.

\section{Classes of partitions}

So far we have not imposed any ex ante restrictions on the set of admissible partitions or jurisdictions. Nevertheless, some special types of partitions play important role in potential applications, and will be of particular interest to our analysis. We then investigate a possibility that the restrictions on partitions we impose are consistent with the stability notions we examine. The most important type of partitions consisting of consecutive (see Greenberg and Weber (1986)) jurisdictions, which are represented by intervals in $I$ (which may or may not contain their endpoints):

Definition 3.1: A partition $P$ of $I$ is consecutive if every $S \in P$ is an interval.

A consecutive partition $P$ can be given by the sequence of intervals $\left\{\left(x_{i-1}, x_{i}\right)\right\}_{1 \leq i \leq n}$, where $0=$ $x_{0}<x_{1}<\ldots x_{n-1}<x_{n}=1$. We will denote the length of an interval $S_{i}=\left(x_{i-1}, x_{i}\right)$ in $P$ by $s_{i}$. Given that $x_{0}=0$ and $x_{n}=1$, a consecutive partition is uniquely defined by the $n-1$ tuple $\left(x_{1}, \ldots, x_{n-1}\right)$, where $0<x_{1}<\ldots x_{n-1}<1$ or by positive numbers $s_{1}, \ldots, s_{n}$, such that $\sum_{i=1}^{n} s_{i}=1$. If no confusion will arise, we use the notation $P=\left(x_{1}, \ldots, x_{n-1}\right)$ or $P=\left(s_{1}, \ldots, s_{n}\right)$. An important property of a consecutive partition $P$ is that a peripheral individual, located on the border of two adjacent intervals in $P$, namely at points $x_{1}, \ldots, x_{n-1}$, will be indifferent between locating being in any of these two jurisdictions. Obviously, this property would be important for the analysis of a potential migration of "almost peripheral" individuals close to the border with other jurisdictions. We will refer to this property as the border indifference: 
Definition 3.2: A consecutive partition $P=\left(x_{1}, \ldots, x_{n-1}\right)$ satisfies border indifference (BI) if for all $i=1, \ldots, n-1$ we have $c\left(x_{i},\left[x_{i-1}, x_{i}\right]\right)=c\left(x_{i},\left[x_{i}, x_{i+1}\right]\right)$.

Note that a partition $P$ satisfies BI if and only if the function $c(\cdot, P)$ is continuous on $I$.

We will also consider a subset of consecutive partitions in which the sequence of jurisdiction sizes is monotone from one endpoint of $I$ to another:

Definition 3.3: A consecutive partition $P$ of $I$ is size-monotone if either $s_{1} \leq s_{2} \leq \ldots \leq s_{n}$ or $s_{1} \geq s_{2} \geq \ldots \geq s_{n}$. (Without loss of generality, we will conduct our analysis for the former case).

If the inequality in Definition 3.3 turns into equality, the partitions with equal-size jurisdictions will arise:

Definition 3.4: A size-monotone partition $P$ of $I$ is homogenous if $s_{1}=\ldots=s_{n}$. A nonhomogeneous partition (not necessarily size-monotone) will be referred to as a heterogenous partition.

We now establish the link between properties of partitions introduced in this section and our stability notions. First,

Proposition 3.5: Any SFM partition is consecutive.

However, this conclusion does not hold for other two stability notions we consider. Moreover, even a consecutive CS (and SAUC) partition may not size-monotone (and thus, not homogeneous):

Proposition 3.6: (i) A CS (and hence a SAUC) partition is not necessarily consecutive.

(ii) A consecutive CS partition is not necessarily size-monotone.

In the next section we provide the characterization results for stable partitions.

\section{Stable Partitions: Characterization Results}

Before proceeding with the characterization of stable consecutive partitions, it would be useful to have a more detailed examination of a monetary cost incurred by members of a jurisdiction in 
the partition. Indeed consider an interval $S=[a, b]$ of length $s=b-a$ and note that the peripheral individuals $a$ and $b$ incur the highest total cost within the jurisdiction. ${ }^{5}$ Their total burden is given by the value of the "peripheral" cost function $\Psi$ defined on $\Re_{+}$:

$$
\Psi(s)=\frac{s}{2}+\frac{g}{s} .
$$

It is easy to see that $\Psi$ is strictly convex, attains its minimum at $d^{*}(g)=\sqrt{2 g}$, and $\min _{s \geq 0} \Psi(s)=$ $\Psi\left(d^{*}(g)\right)=d^{*}(g)$. Moreover, it decreases for $s<d^{*}(g)$, increases for $s>d^{*}(g)$, and attains any value $d>d^{*}(g)$ twice (once at some $s<d^{*}(g)$, and once at some $s^{\prime}>d^{*}(g)$ ). Thus, $s=d^{*}(g)$ is the "optimal size" of an interval jurisdiction that minimizes the cost of the most disadvantaged individuals. Note also that the border indifference condition BI can be presented as $\Psi\left(s_{1}\right)=\ldots=$ $\Psi\left(s_{n}\right)$. Hence, any partition which satisfies BI contains jurisdictions of at most two distinct sizes.

We now provide the complete characterization of SFM partitions. In the multi-jurisdictional case we determine the bounds for jurisdictional sizes in a SFM partition:

Proposition 4.1: (i) A consecutive heterogeneous partition $P$ into multiple jurisdictions is SFM if and only if it satisfies BI, and for each $S \in P$ we have $|S| \in\left[\frac{d^{*}(g)}{\sqrt{2}}, \sqrt{2} d^{*}(g)\right]$;

(ii) A homogenous partition $P$ into multiple interval jurisdictions of size $s$ is SFM if and only if $s \geq \frac{d^{*}(g)}{\sqrt{2}}$;

(iii) The grand jurisdiction $I$ is always SFM.

Given the fact that a heterogenous partition under BI can contain jurisdictions of different sizes, in order to verify whether this partition is SFM, one has to check whether the jurisdictions are neither too small nor too large. In the homogenous case the verification of only one bound would suffice.

It is worthwhile to establish the link between SFM and CS (to recall both notions are weaker than SAUC). It turns out that, in heterogenous case, SFM is weaker than CS. Even more surprising

\footnotetext{
${ }^{5}$ In the case of closed intervals, two jurisdictions have a common peripheral individual, that could be considered to be a member of both, whereas in the case of open intervals there could be an individual that belongs to no jurisdiction. We discuss this technical point in the Appendix A.
} 
is the fact that the inclusion is reverse in the homogenous case, where the set of CS partitions is a subset of the SFM set:

Proposition 4.2: (i) Every heterogenous SFM partition is CS.

(ii) Every consecutive homogenous CS partition is SFM.

We now turn to the examination of SAUC and CS partitions. Even though Proposition 3.6 indicates that SAUC and CS partitions could be non-consecutive, in what follows we concentrate on the case when only admissible jurisdictions are intervals. Proposition 3.6 also implies if a CS (SAUC) partition is consecutive, it need not be size-monotone. However, once size-monotonicity is imposed, ${ }^{6}$ we obtain sufficient conditions for SAUC and CS:

Proposition 4.3: Let $P=\left(s_{1}, \ldots, s_{n}\right)$ be a size-monotone partition of $I$ where $d^{*}(g) \leq s_{1}$. Then $P$ satisfies BI and is SAUC.

Proposition 4.4: Let $P=\left(s_{1}, \ldots, s_{n}\right)$ be a size-monotone partition of $I$ where $d^{*}(g) \leq s_{1} \leq \ldots \leq$ $s_{n} \leq \sqrt{2} d^{*}(g)$. Then $P$ satisfies BI and is CS.

To guarantee that a size-monotone partition is SAUC, it is enough to verify that jurisdictions in this partition are not too small: the smallest jurisdiction should not be smaller then the "optimal" size $d^{*}(g)$. If we wish to impose a stricter requirement of CS, we also have to limit the size of jurisdictions from above so that the size of the largest jurisdiction does not exceed $\sqrt{2} d(g)$. These sufficient conditions allow a wide range of stable structures.

If we further restrict our attention to homogenous partitions, we obtain a necessary and sufficient condition for CS:

Proposition 4.5: (i) A homogenous partition of $I$ which consists of multiple jurisdictions of size $s$ (and therefore, satisfies BI) is CS if and only if $\frac{d^{*}(g)}{\sqrt{2}} \leq s \leq d^{*}(g)(1+\sqrt{2})$.

(ii) The interval $I$ is CS if and only if $\sqrt{g} \geq \frac{1}{2+\sqrt{6}} \cdot{ }^{7}$

\footnotetext{
${ }^{6}$ To recall, we restrict our examination to the case $s_{1} \leq \ldots \leq s_{n}$.

${ }^{7}$ Note that this condition also gives a necessary and sufficient conditions for (core) stability of a given jurisdiction $S_{i} \in P$ against internal threats, i.e., against potentially seceding jurisdictions $S \subset S_{i}$.
} 
Since $s=\frac{1}{n}$ and $d^{*}(g)=\sqrt{2 g}$, last proposition allows us to determine the values of $n$, which, for given $g>0$, admit CS $n$-partitions. Namely,

if $\sqrt{g} \geq \frac{1}{2+\sqrt{6}}$, then 1-partition is CS;

if $\sqrt{g} \leq \frac{1}{n} \leq \sqrt{g}(2+\sqrt{2})$, where $n>1$, then there exists a CS $n$-partition.

We also provide an alternative version (actually a corollary) of Proposition 4.5 by identifying, for given $n$, the range of values of $g$ that yield a homogenous CS $n$-partition.

Proposition 4.6: (i) A multi-jurisdictional homogenous n-partition is CS if and only if

$$
\frac{1}{n^{2}[2+\sqrt{2}]^{2}} \leq g \leq \frac{1}{n^{2}}
$$

(ii) A homogenous CS partition exists for any $g>0$.

\section{$5 \quad$ Brief Concluding Remarks}

In this paper we examined a population represented by a continuum of individuals uniformly distributed over the unit interval that faces a problem of location and financing of public facilities. Under the assumption of equal share, we consider various notions of stability of jurisdiction structures (stability under unanimous consent, free mobility and core) and provide a characterization of stable structures.

This leaves a room for extensions of those results. One can consider a more general type of distribution function alternative (e.g., monotone density functions in Haeringer (2001), logconcave distributions (Haimanko et al. (2005), the general case in Haimanko et al. (2004)). Even more challenging and open area of research is a possible extension of the set of feasible locations to a multidimensional setting. Indeed, a multidimensional set of alternatives is an appropriate framework for a large array of applications in economics, political science or geography, where preferences or locations of an individual are characterized by more than one parameter. We hope that a progress can be achieved along this line of research. 


\section{Appendix A - Preliminary Results}

We start by introducing some notation. For every jurisdiction $S$ (either a member of an initial jurisdiction structure, or a potentially deviating group), denote by $l(S)=\inf \{t \mid t \in S\}$ and $r(S)=$ $\sup \{t \mid t \in S\}$ its peripheral individuals. We will assume for every $S$ both $l(S)$ and $r(S)$ belong to $S$. Thus, if two jurisdictions have a common peripheral individual, she is considered a member of both. We will ignore a finite number of such individuals (at most $n$ as the number of jurisdictions in the partition). ${ }^{8}$

We will use the notation $c(t, S)$ even if an individual $t$ does not belong to $S$. In this case, $c(t, S)$ stands for the total cost $c(t, S \cup\{t\})$ an individual $t$ would incur if she joins jurisdiction $S$. Note that, given $S, c(t, S)=c(t, S \cup\{t\})=|t-m(S)|+\frac{g}{|S|}$ is a continuous single-dipped function. For any partition $P$, any group $S$, and any individual $t \in I$, we define $\Delta(t, S, P)=c(t, S)-c(t, P)$. Recall that, whenever a group $S$ is allowed to deviate (under either SAUC, CS or SFM), all members of $S$ must be strictly better off relatively to their cost levels in the original partition $P$. Hence, $S$ would deviate only if $\Delta(t, S, P)<0$ for all $t \in S$.

Let $g>0$ be given and we will use the notation $d^{*}$ instead of $d^{*}(g)$ for the optimal size of jurisdiction given. We need the following remarks and lemmas.

Remark A.1 The peripheral cost function $\Psi(s)=\frac{s}{2}+\frac{g}{s}$, defined in Section 4, satisfies the following conditions:

$$
\begin{gathered}
\frac{d \Psi(s)}{d s} \in\left[-\frac{1}{2}, \frac{1}{2}\right] \quad \text { for } s \in[\sqrt{g},+\infty) . \\
\Psi(s+a)>\Psi(s)-\frac{|a|}{2} \text { for } s \in[\sqrt{g},+\infty) \text { and } a>-s .
\end{gathered}
$$

This follows from direct differentiation and the fact that the convexity of $\Psi$ implies $\Psi(s+a)>$ $\Psi(s)+\Psi^{\prime}(s) a$.

Remark A.2. If $P$ is SAUC, then for any $S_{i}, S_{j} \in P, i \neq j$ we have $m\left(S_{i}\right) \neq m\left(S_{j}\right)$.

The proof is straightforward.

\footnotetext{
${ }^{8}$ All the statements in the paper remain true without this assumption, but its absence would make the presentation unnecessarily burdened by technical details. The proofs in absence of this assumption are available from authors upon request.
} 
Remark A.3. For any $S$ we have $\min \{c(l(S), S), c(r(S), S)\} \geq \Psi(|S|) \geq d^{*}$ and $\max \{c(l(S), S), c(r(S), S)\}=\max \{c(t, S): t \in S\} \geq \Psi(|r(S)-l(S)|)$.

All inequalities are straightforward except the last one. Assume $c(l(S), S) \geq c(r(S), S)$. Then $m(S) \geq m([l(S), r(S)])$, and

$$
c(l(S), S)=|l(S)-m(S)|+\frac{g}{|S|} \geq|l(S)-m([l(S), r(S)])|+\frac{g}{|r(S)-l(S)|}=\Psi(|r(S)-l(S)|) .
$$

Remark A.4. If $t$ is a peripheral individual in $S$, then $c(t, S) \geq \min _{s} \Psi(s)=d^{*}=\sqrt{2 g}>1.4 \sqrt{g}$. If $S$ is a group that violates SAUC of the jurisdiction structure $P$, then $c(t, P)>d^{*}$, and hence $\left|S^{t}\right| \neq d^{*}$

Remark A.5. Let $P=\left(s_{1}, \ldots, s_{n}\right)$ be a consecutive partition, with $s_{i} \in[\sqrt{g}, 2 \sqrt{g}]$. Then for any $t \in I$ we have

$$
c(t, P) \leq \max _{1 \leq i \leq n}\left\{\Psi\left(s_{i}\right)\right\} \leq \max \{\Psi(s): s \in[\sqrt{g}, 2 \sqrt{g}]\}=\Psi(\sqrt{g})=\Psi(2 \sqrt{g})=1.5 \sqrt{g} .
$$

Lemma A.6. Let $P=\left(x_{1}, \ldots, x_{n-1}\right)$ be a consecutive partition, with $s_{i} \in[\sqrt{g}, 2 \sqrt{g}]$. If a group $S$ violates CS of $P$, then there exist $i, j$ such that $\max \left\{\left|l(S)-x_{i}\right|,\left|r(S)-x_{j}\right|\right\}<0.1 \sqrt{g}$.

Proof: We can assume $l(S) \in S_{i}=\left[x_{i-1}, x_{i}\right] \in P$ and $l(S) \geq m\left(S_{i}\right)$. Then:

$$
c\left(x_{i}, S_{i}\right)-c(l(S), P)=c\left(x_{i}, S_{i}\right)-c\left(l(S), S_{i}\right)=\left|x_{i}-m\left(S_{i}\right)\right|-\left|l(S)-m\left(S_{i}\right)\right|=\left|l(S)-x_{i}\right| .
$$

Then Remarks A.5 and A.4 and the fact that $S$ violates CS of $P$ yield

$$
1.4 \sqrt{g} \leq c(l(S), S)<c(l(S), P) \leq 1.5 \sqrt{g}-\left|l(S)-x_{i}\right|
$$

, and, hence, $\left|l(S)-x_{i}\right|<0.1 \sqrt{g}$. Since the similar argument works for an evaluation of the distance $\left|r(S)-x_{j}\right|$, the conclusion follows.

Lemma A.7. Let $P$ be a consecutive partition, satisfying BI. Then for every jurisdiction $S$, the function $\Delta(\cdot, S, P)$ is a continuous (in fact, a peace-wise linear) function on $I$, single-dipped, and attains its minimum at $m(S)$.

Proof: Continuity follows from BI. For $t \leq m(S)$ we have

$$
\begin{aligned}
\Delta(t, S, P) & =c(t, S)-c(t, P)=|t-m(S)|-\left|t-m\left(S^{t}\right)\right|+\frac{g}{|S|}-\frac{g}{\left|S^{t}\right|} \\
& =m(S)-t-\left|t-m\left(S^{t}\right)\right|+C_{1}\left(S^{t}\right)= \begin{cases}C_{2}\left(S^{t}\right), & t \leq m\left(S_{i}\right) \\
C_{2}\left(S^{t}\right)-2 t, & t \geq m\left(S_{i}\right) .\end{cases}
\end{aligned}
$$


Here $C_{1}\left(S^{t}\right), C_{2}\left(S^{t}\right)$ are constant on each $S^{t}$. Hence, $\Delta(t, S, P)$ is (non-strictly) decreasing on each interval $S^{t} \cap[0, m(S)]$. Thus, it is decreasing on $[0, m(S)]$. Analogously, it is increasing on $[m(S), 1]$.

Lemma A.8. Let $P$ be a consecutive partition, satisfying BI. If no interval can violate CS of $P$, then $P$ is CS.

Proof Assume that $S$ violate CS of $P$ by deviating from $P$ and making all its members better off. Then $\Delta(l(S), S, P)<0$ and $\Delta(r(S), S, P)<0$, and, hence, by Lemma A.7, $\Delta(t, S, P)<0$ on the entire interval $[l(S), r(S)]$. But then the interval $\left[m(S)-\frac{|S|}{2}, m(S)+\frac{|S|}{2}\right]$ can also make all its members better off, a contradiction.

We now turn to the proof of our main results.

\section{Appendix B - Main Results}

Proof of Proposition 3.5. Assume to the contrary, that $P$ is SFM but is not consecutive. Then there are two different jurisdictions $S, S^{\prime} \in P$ and individuals $t_{1}, t_{2} \in S, t^{\prime} \in S^{\prime}$, such that $t_{1}<t^{\prime}<t_{2}$. Without loss of generality, we can assume $m(S)<m\left(S^{\prime}\right)$ (see Remark A.2). We have for all $t \in I$ :

$$
\begin{gathered}
c(t, S)-c\left(t, S^{\prime}\right)=|t-m(S)|-\left|t-m\left(S^{\prime}\right)\right|+\frac{g}{|S|}-\frac{g}{\left|S^{\prime}\right|} \\
=|t-m(S)|-\left|t-m\left(S^{\prime}\right)\right|+C= \begin{cases}m(S)-m\left(S^{\prime}\right)+C, & t \leq m(S) ; \\
2 t-m(S)-m\left(S^{\prime}\right)+C, & m(S) \leq t \leq m\left(S^{\prime}\right) ; \\
m\left(S^{\prime}\right)-m(S)+C, & m\left(S^{\prime}\right) \leq t .\end{cases}
\end{gathered}
$$

Hence, $c(t, S)-c\left(t, S^{\prime}\right)$ is an increasing function of $t$ on $[0,1]$, and so $c\left(t_{1}, S\right)-c\left(t_{1}, S^{\prime}\right)<c\left(t^{\prime}, S\right)-$ $c\left(t^{\prime}, S^{\prime}\right)<c\left(t_{2}, S\right)-c\left(t_{2}, S^{\prime}\right)$. But SFM implies that no individual can improve her fate by changing jurisdiction. In particular, we should have $c\left(t_{1}, S\right) \leq c\left(t_{1}, S^{\prime}\right), c\left(t_{2}, S\right) \leq c\left(t_{2}, S^{\prime}\right)$, and $c\left(t^{\prime}, S^{\prime}\right) \leq c\left(t^{\prime}, S\right)$, which contradicts the above inequalities.

Proof of Proposition 3.6. (i) Let $g=\frac{1}{18}$ and therefore $d^{*}=\frac{1}{3}$. Consider $P$ that consists of three jurisdictions, $S_{1}=\left[0, d^{*}-\varepsilon\right] \bigcup[1-\varepsilon, 1], S_{2}=\left(d^{*}-\varepsilon, 2 d^{*}-\varepsilon\right), S_{3}=\left(2 d^{*}-\varepsilon, 1-\varepsilon\right)$. Assume, in negation that a jurisdiction $S$ can deviate from $P$ and to violate CS of $P$. By Remark 
A.4, neither $l(S)$ nor $r(S)$ can belong to either $S_{2}, S_{3}$ or $[0, d-\varepsilon]$, since any individual $t$ located in one of those areas has $c(t, P) \leq d$. Hence, $S \subset[1-\varepsilon, 1] \subset S_{1}$.

Note that for any $t \in I$ we have $c(t, P)=\left|t-m\left(S^{t}\right)\right|+\frac{g}{d^{*}} \leq 1+\frac{g}{d^{*}}=\frac{7}{6}$. So, if $S$ deviates, it has to be $c(t, S)<c(t, P) \leq \frac{7}{6}$ for all $t \in S$. But, for $\varepsilon$ small enough, $c(t, S) \geq \frac{g}{|S|}=\frac{1}{18 \varepsilon} \geq \frac{7}{6}$, a contradiction.

(ii) Let $g=\frac{1}{18}$ and $d^{*}=\frac{\sqrt{2}}{6}$. Consider a consecutive 4-partition $P=\left(s_{1}, s_{2}, s_{3}, s_{4}\right)$, where $s_{1}=s_{4}=\frac{1}{6}$ and $s_{2}=s_{3}=\frac{1}{3}$. By Proposition 4.1, part (i), it is SFM, and by Proposition 4.2, part (i), it is CS, too. However, $P$ is obviously not size-monotone.

Proof of Proposition 4.1 (i) "Only if" part. Let $P=\left\{S_{1}, \ldots, S_{n}\right\}=\left(x_{1}, \ldots, x_{n-1}\right)=$ $\left(s_{1}, \ldots, s_{n}\right)$ be a consecutive heterogeneous SFM $n$-partition, $n>1$.

Assume that BI condition does not hold. Then, without loss of generality, there exists an individual $x_{i}, i \in\{1, \ldots, n-1\}$, peripheral for $S_{i}$ and $S_{i+1}$, such that $c\left(x_{i}, S_{i}\right)>c\left(x_{i}, S_{i+1}\right)$. But then for any small enough positive $\varepsilon$ we have $c\left(x_{i}-\varepsilon, S_{i}\right)>c\left(x_{i}-\varepsilon, S_{i+1}\right)$, so the interval $\left(x_{i}-\varepsilon, x_{i}\right)$ would benefit from migrating to $S_{i+1}$, which contradicts SFM.

Now, since $P$ satisfies BI and is heterogeneous, it contains jurisdictions of exactly two sizes, $s^{\prime}$ and $s^{\prime \prime}$, where $s^{\prime}<s^{\prime \prime}$ and $\Psi\left(s^{\prime}\right)=\Psi\left(s^{\prime \prime}\right)$. To complete this part, it is enough to check that $s^{\prime} \geq \sqrt{g}$ (since $\Psi(\sqrt{g})=\Psi(2 \sqrt{g}), s^{\prime \prime} \leq 2 \sqrt{g}$ follows).

Assume to the contrary that $s^{\prime}<\sqrt{g}$. Then we have $s^{\prime \prime}>2 \sqrt{g}>2 s^{\prime}$ and $s^{\prime}<\frac{g}{s^{\prime}}$. We can assume without loss of generality that $S_{1}=\left[0, s^{\prime}\right]$ and $S_{2}=\left[s^{\prime}, s^{\prime}+s^{\prime \prime}\right]$. We check that the group $T=\left[s^{\prime \prime}-\varepsilon, s^{\prime}+s^{\prime \prime}\right] \subset S_{2}$ would benefit from joining $S_{1}=\left[0, s^{\prime}\right]$, for $\varepsilon$ such that $0<\varepsilon<\left(s^{\prime \prime}-2 s^{\prime}\right) / 2$ and $\varepsilon<\Psi\left(s^{\prime \prime}\right)-\Psi\left(2 s^{\prime}+\varepsilon\right)$ (such $\varepsilon>0$ exists, since $\Psi\left(s^{\prime \prime}\right)>\Psi\left(2 s^{\prime}\right)$ and $\Psi$ is continuous).

First, note that $|T|=s^{\prime}+\varepsilon>s^{\prime}$, so $m\left(T \cup S_{1}\right) \in T$; moreover, $m\left(T \cup S_{1}\right)=s^{\prime \prime}-\frac{\varepsilon}{2}>s^{\prime \prime}-\varepsilon>$ $m\left(S_{2}\right)=s^{\prime}+\frac{s^{\prime \prime}}{2}$. We will now check that $\Delta\left(t, T \cup S_{1}, P\right)=c\left(t, T \cup S_{1}\right)-c(t, P)<0$ for all $t \in T$. First,

$$
\Delta\left(s^{\prime}+s^{\prime \prime}, T \cup S_{1}, P\right)=c\left(s^{\prime}+s^{\prime \prime}, T \cup S_{1}\right)-c\left(s^{\prime}+s^{\prime \prime}, P\right)=\Psi\left(2 s^{\prime}+\varepsilon\right)-\Psi\left(s^{\prime \prime}\right)<-\varepsilon<0 .
$$


Next, for $t \in T$ with $m\left(S_{2}\right)<m\left(T \cup S_{1}\right)=s^{\prime \prime}-\frac{\varepsilon}{2} \leq t \leq s^{\prime}+s^{\prime \prime}$, we have

$$
\Delta\left(t, T \cup S_{1}, P\right)=c\left(t, T \cup S_{1}\right)-c(t, P)=\Delta\left(s^{\prime}+s^{\prime \prime}, T \cup S_{1}, P\right)=\Psi\left(2 s^{\prime}+\varepsilon\right)-\Psi\left(s^{\prime \prime}\right)<-\varepsilon<0 .
$$

Finally, for $t \in T$ with $m\left(S_{2}\right)<s^{\prime \prime}-\varepsilon \leq t \leq s^{\prime \prime}-\frac{\varepsilon}{2}=m\left(T \cup S_{1}\right)$, we have

$$
\begin{aligned}
& \Delta\left(t, T \cup S_{1}, P\right)=\Delta\left(s^{\prime}+s^{\prime \prime}, T \cup S_{1}, P\right)+2\left(m\left(T \cup S_{1}\right)-t\right)= \\
& \Psi\left(2 s^{\prime}+\varepsilon\right)-\Psi\left(s^{\prime \prime}\right)+\left(2 s^{\prime \prime}-\varepsilon-2 t\right)<2\left(s^{\prime \prime}-\varepsilon-t\right) \leq 0 .
\end{aligned}
$$

Thus, $T$ would benefit from deviation, which contradicts SFM.

Proof of Proposition 4.1 (ii). "Only if" part. Let $P$ be a homogenous partition into intervals of size $s$. If $s<\sqrt{g}$, then $s<\frac{g}{s}$, and a jurisdiction $S_{1}=[0, s]$ would benefit from joining $S_{2}=[s, 2 s]$. Indeed, for any $t \in S_{1}$ we have

$$
c\left(t, S_{1}\right)=\left|t-\frac{s}{2}\right|+\frac{g}{s} \geq|t-s|-\left|\frac{s}{2}-s\right|+\frac{g}{s}=|t-s|+\frac{g}{s}-\frac{s}{2}>|t-s|+\frac{g}{2 s}=c\left(t, S_{1} \cup S_{2}\right) .
$$

Note that in this case all members of $S_{1} \cup S_{2}$ benefit form joining together. Thus, for $s<\sqrt{g}$ partition $P$ is neither SFM nor CS.

We will use Proposition 4.2 to prove the "if" part of Proposition 4.1 (i) and (ii), so we prove Proposition 4.2 before completing the proof of Proposition 4.1.

Proof of Proposition 4.2. part (i) The "only if" part of Proposition 4.1 (i) implies that if a heterogeneous $P$ is SFM then it satisfies BI and consists of intervals of two different sizes, $s^{\prime}$ and $s^{\prime \prime}$, where $\sqrt{g} \leq s<s^{\prime \prime} \leq 2 \sqrt{g}$ and $\Psi\left(s^{\prime}\right)=\Psi\left(s^{\prime \prime}\right)=\psi \leq 1.5 \sqrt{g}$. Proposition 4.2 follows from the following stronger statement:

Proposition B.1: If a consecutive partition $P$ satisfies BI and $\sqrt{g} \leq\left|S_{i}\right| \leq 2 \sqrt{g}$ for all jurisdictions $S_{i} \in P$, then $P$ is CS.

Proof: If $P$ satisfies the conditions of Proposition B.1, then it satisfies BI and hence consists of intervals of at most two sizes, $s^{\prime}$ and $s^{\prime \prime}$, where $\sqrt{g} \leq s^{\prime} \leq s^{\prime \prime} \leq 2 \sqrt{g}$ and $\Psi\left(s^{\prime}\right)=\Psi\left(s^{\prime \prime}\right)=\psi \leq$ $1.5 \sqrt{g}$. 
Hence, by Remark A.5, we have $c(t, P) \leq 1.5 \sqrt{g}$ for any $t \in P$. If a jurisdiction $S$ can deviate under CS, then, by Lemma A.8, we may assume it is an interval. Let $p$ be a peripheral individual in $S$. Then Remark A.3 yields $\Psi(|S|) \leq c(p, S)<c(p, P) \leq 1.5 \sqrt{g}$, and, therefore, $|S| \in(\sqrt{g}, 2 \sqrt{g})$.

By Lemma A.6 we can find $x_{i}$ and $x_{j}$, peripheral individuals in jurisdictions in $P$, such that that both differences $a_{1}=\left|l(S)-x_{i}\right|$ and $a_{2}=\left|r(S)-x_{j}\right|$ are smaller than $0.1 \sqrt{g}$. Without loss of generality, assume that $a_{1} \geq a_{2}$. We have $S=[l(S), r(S)]=\left[x_{i} \pm a_{1}, x_{j} \pm a_{2}\right]$ and $i \leq j$. We now consider the following four possible cases.

CASE 1: $i=j$. Here $|S|=r(S)-l(S) \leq\left|l(S)-x_{i}\right|+\left|r(S)-x_{i}\right| \leq 0.2 \sqrt{g}$, which contradicts the assumption that $|S|>\sqrt{g}$.

CASE $2: i=j-1$, so $\left[x_{j-1}, x_{j}\right]=S_{j} \in P$. Then, given $a_{1} \geq a_{2}$, and Remark A.1, we have:

$$
c(l(S), S)=\Psi(|S|)=\Psi\left(s_{j} \pm a_{1} \pm a_{2}\right)>\Psi\left(s_{j}\right)-\frac{\left| \pm a_{1} \pm a_{2}\right|}{2} \geq \psi-\left|a_{1}\right|=c(l(S), P),
$$

a contradiction to $S$ being a deviating group.

CASE 3: $i=j-2$. Then, since $\Psi$ is increasing on $[\sqrt{2 g},+\infty)$, we have:

$$
\begin{gathered}
c(l(S), S)=\Psi(|S|)=\Psi\left(s_{j}+s_{j-1} \pm a_{1} \pm a_{2}\right)>\Psi\left(s_{j}+s_{j-1}\right)-\frac{\left| \pm a_{1} \pm a_{2}\right|}{2} \geq \\
\Psi(2 \sqrt{g})-\left|a_{1}\right|=1.5 \sqrt{g}-\left|a_{1}\right| \geq \psi-\left|a_{1}\right|=c(l(S), P),
\end{gathered}
$$

a contradiction again. Finally,

CASE $4: j-i \geq 3$. Then, $|S| \geq 3 \sqrt{g}-0.2 \sqrt{g}=2.8 \sqrt{g}>2 \sqrt{g}$, a contradiction again. This completes the proof of both Proposition B.1 and part (i) of Proposition 4.2. Part (ii) of the latter proposition follows immediately from Proposition 4.5.

Proof of Proposition 4.1 (i). "If" part. Again, we will prove a bit stronger statement, namely:

Proposition B.2: If a consecutive partition $P$ satisfies BI and $\sqrt{g} \leq\left|S_{i}\right| \leq 2 \sqrt{g}$ for all jurisdictions $S_{i} \in P$, then $P$ is SFM.

Proof: Consider a consecutive $P$, which satisfies BI and hence consists of intervals of at most two 
sizes, $s^{\prime}$ and $s^{\prime \prime}$, where by the assumption $\sqrt{g} \leq s^{\prime} \leq s^{\prime \prime} \leq 2 \sqrt{g}$ and so $\Psi\left(s^{\prime}\right)=\Psi\left(s^{\prime \prime}\right)=\psi \leq 1.5 \sqrt{g}$. Proposition B.1 guarantees that $P$ is CS.

Assume that $P$ is not SFM. Hence, there exists a group $T$, and a jurisdiction $S_{i} \in P$ such that all members of $T$ prefer $T \cup S_{i}$ to $T$. We can choose $T$ so that $T \cap S_{i}=\emptyset$. We partition $T$ into $T_{l}$ and $T_{r}$, which lie respectively to the left and to the right of $S_{i}=\left[x_{i-1}, x_{i}\right]$ : we have $t \leq x_{i-1}$ for all $t \in T_{l}$, and $t \geq x_{i}$ for all $t \in T_{r}$.

First, one of $T_{l}$ and $T_{r}$ should be empty. Indeed, let $T^{\prime}=T \cup S_{i}$ with $l\left(T^{\prime}\right) \in T_{l}, r\left(T^{\prime}\right) \in$ $T_{r}$. Since both values $\Delta\left(l\left(T^{\prime}\right), T^{\prime}, P\right)$ and $\Delta\left(r\left(T^{\prime}\right), T^{\prime}, P\right)$ are negative, Lemma A.7 implies that $\Delta\left(t, T^{\prime}, P\right)<0$ for all $t \in\left[l\left(T^{\prime}\right), r\left(T^{\prime}\right)\right]$. But this means that the group $T^{\prime}$ can deviate by forming its own jurisdiction, a contradiction to the fact that $P$ is CS.

Now, without loss of generality, assume $T_{r}=\emptyset$ and $T=T_{l}, T^{\prime}=\left[l\left(T^{\prime}\right), x_{i}\right]$. By Remark A.5 we have $1.5 \sqrt{g} \geq c(l(S), P)>c\left(l(S), T^{\prime}\right) \geq \Psi\left(\left|T^{\prime}\right|\right)$, and so $\left|T^{\prime}\right|<2 \sqrt{g}$. Since $\left|S_{i}\right| \geq \sqrt{g}$, we obtain $|T|<\sqrt{g}$ and so $m\left(T^{\prime}\right) \in S_{i}$.

Next, note that that the individual located at $m\left(T^{\prime}\right)$ is better off at $T^{\prime}$ than at $S_{i}$ : her tax contribution declines since jurisdiction becomes larger, and her transportation cost drops to zero. Thus, $\Delta\left(l\left(T^{\prime}\right), T^{\prime}, P\right)<0, \Delta\left(m\left(T^{\prime}\right), T^{\prime}, P\right)<0$, and Lemma A.7 implies that $\Delta\left(t, T^{\prime}, P\right)<0$ for all $t \in\left[l\left(T^{\prime}\right), m\left(T^{\prime}\right)\right]$. It tells us that the interval $T^{\prime \prime}=\left[x_{i-1}-|T|, x_{i-1}\right]=\left[p, x_{i-1}\right]$, with $\left|T^{\prime \prime}\right|=|T|<\sqrt{g}$, also can deviate under SFM by joining the adjacent interval $S_{i}$.

If $\left|S_{i}\right|=s^{\prime \prime}$, then $c\left(p, S_{i} \cup T^{\prime \prime}\right)=\Psi\left(\left|S_{i} \cup T^{\prime \prime}\right|\right)>\Psi\left(s^{\prime \prime}\right)=\Psi\left(s^{\prime}\right) \geq c(l(S), P)$, which contradicts the assumption that $T^{\prime \prime}$ can deviate under SFM. Hence, $\left|S_{i}\right|=s^{\prime}$.

Furthermore, we have $\left|T^{\prime \prime}\right|<\sqrt{g}$, and its left endpoint belongs to $S_{i-1}$. From Remark A.1 we obtain: if $p \geq m\left(S_{i-1}\right)$, then

$$
c\left(p, T^{\prime \prime} \cup S_{i}\right)=\Psi\left(\left|T^{\prime \prime} \cup S_{i}\right|\right)=\Psi\left(s^{\prime}+\left|T^{\prime \prime}\right|\right)>\Psi\left(s^{\prime}\right)-\left|T^{\prime \prime}\right|=\psi-\left|T^{\prime \prime}\right|=c\left(p, S_{i-1}\right)=c(p, P) ;
$$

if $p \leq m\left(S_{i-1}\right)$, then also

$$
\begin{gathered}
c\left(p, T^{\prime \prime} \cup S_{i}\right)=\Psi\left(\left|T^{\prime \prime} \cup S_{i}\right|\right)=\Psi\left(s^{\prime}+s_{i-1}-\left(s_{i-1}-|T|\right)\right)>\Psi\left(s^{\prime}+s_{i-1}\right)-\left(s_{i-1}-|T|\right) \\
\Psi(2 \sqrt{g})-\left(s_{i-1}-|T|\right)=\psi-\left(s_{i-1}-|T|\right)=c\left(p, S_{i-1}\right)=c(p, P) .
\end{gathered}
$$


Hence, both possibilities contradict the assumption that $T^{\prime \prime}$ can deviate by joining $S_{i}$ under SFM. This completes the proof of both Proposition B.2 and Proposition 4.1 (i).

Proof of Proposition 4.1 (ii). "If" part. Let $P$ be a homogenous partition into intervals of size $s \geq \sqrt{g}$. If $\sqrt{g} \leq s \leq \sqrt{2 g}$, then SFM follows from Proposition B.2 above. Let $s \geq \sqrt{2 g}$. Suppose that a group $T$ contemplates joining a jurisdiction $S_{i}=[(i-1) s, i s] \in P$. By Remark A.1, we have:

$$
c\left(l(S), T \cup S_{i}\right) \geq \Psi\left(\left|T \cup S_{i}\right|\right) \geq \Psi(s) \geq c(l(S), P),
$$

and the individual at $l(S)$ would not benefit from this migration. Thus, there are no profitable migrations, and $P$ is SFM.

Proof of Proposition 4.3. Let $P=\left\{S_{1}, \ldots, S_{n}\right\}=\left(s_{1}, \ldots, s_{n}\right)$ be a size-monotone partition, with $d=\sqrt{2 g} \leq s_{1} \leq \ldots \leq s_{n}$. If it is not SAUC, then there exists $T \subset I$ and $S_{i} \in P$, such that $S=T \cup S_{i}$ is preferred to $P$ by all its members.

Let $S$ be such that $l(S) \in S_{j} \in P, j \leq i$. Using Remarks A.1 and A.3, we obtain $c(l(S), P)=$ $c\left(l(S), S_{j}\right) \leq \Psi\left(s_{j}\right) \leq \Psi\left(s_{i}\right)<\Psi(|S|) \leq c(l(S), S)$, a contradiction.

Proof of Proposition 4.4. Let $P=\left\{S_{1}, \ldots, S_{n}\right\}=\left(s_{1}, \ldots, s_{n}\right)=\left(x_{1}, \ldots, x_{n-1}\right)$ be a sizemonotone partition, with $d=\sqrt{2 g} \leq s_{1} \leq \ldots \leq s_{n} \leq 2 \sqrt{g}$. By Remark A.5, $c(t, P) \leq 1.5 \sqrt{g}$ for all $t \in I$. If $P$ is not CS, then there exists a group $T \subset I$ which would be better of by forming its own jurisdiction. By Lemma A.6, there exist $i$ and $j$, such that both differences $a_{1}=\left|l(S)-x_{i}\right|$ and $a_{2}=\left|r(S)-x_{j}\right|$ are smaller than $0.1 \sqrt{g}$.

Since $s_{k} \geq \sqrt{2 g}$ for all $S_{k} \in P$, we have $i \leq j$.

If $i=j$, we get $|T|<0.2 \sqrt{g}$ and $c(l(S), T) \geq \frac{g}{|T|}>1.5 \sqrt{g} \geq c(l(S), P)$.

If $i<j-1$, we get $|T|>1.8 \sqrt{2 g}$ and $c(l(S), T)>\Psi(1.8 \sqrt{2 g})>1.5 \sqrt{g} \geq c(l(S), P)$.

Both cases contradict the fact that $T$ can beneficially deviate under CS, hence, $i=j-1$. This means that the individuals $x_{i}=x_{j-1}$ and $x_{j}$ are peripheral in $S_{j} \in P$. Thus, $r(S)-l(S) \geq$ 
$s_{j}-0.2 \sqrt{g} \geq \sqrt{2 g}-0.2 \sqrt{g}=(\sqrt{2}-0.2) \sqrt{g}$.

Consider two possible cases:

CASE 1: $|T|<\left|S_{j}\right|=s_{j}$. Then $c\left(m\left(S_{j}\right), P\right)<c\left(m\left(S_{j}\right), T\right)$ (the contribution of $m\left(S_{j}\right)$ is larger in $T$, while her transportation cost is smaller (zero) at $P$ ), hence $m\left(S_{j}\right) \notin T$. For the same reason, the individuals from the half of $S_{j}$ between $m\left(S_{j}\right)$ and its peripheral individual, which does not contain $m(T)$ in its interior, cannot belong to $T$. This observation yields $|T| \leq \frac{s_{j}}{2}+0.2 \sqrt{g} \leq 1.2 \sqrt{g}$. Moreover,

$$
\begin{aligned}
& \max \{c(l(S), T), c(r(S), T)\} \geq \frac{|r(S)-l(S)|}{2}+\frac{g}{|T|} \geq \frac{1}{2}\left(s_{j}-0.2 \sqrt{g}\right)+\frac{g}{\frac{s_{j}}{2}+0.2 \sqrt{g}}= \\
& \left(\frac{s_{j}}{4}-0.2 \sqrt{g}\right)+\frac{1}{2}\left(\frac{s_{j}}{2}+0.2 \sqrt{g}\right)+\frac{g}{\frac{s_{j}}{2}+0.2 \sqrt{g}}=\left(\frac{s_{j}}{4}-0.2 \sqrt{g}\right)+\Psi\left(\frac{s_{j}}{2}+0.2 \sqrt{g}\right) .
\end{aligned}
$$

Since $\sqrt{2 g} \leq s_{j} \leq 2 \sqrt{g}$, we have $\frac{s_{j}}{2}+0.2 \sqrt{g} \leq 1.2 \sqrt{g}<d^{*}$, and, given that $\Psi$ decreases on $\left[0, d^{*}\right]$, we obtain $\Psi\left(\frac{s_{j}}{2}+0.2 \sqrt{g}\right) \geq \Psi(1.2 \sqrt{g})$. Thus, we can continue:

$$
\begin{aligned}
& \max \{c(l(S), T), c(r(S), T)\} \geq\left(\frac{\sqrt{2 g}}{4}-0.2 \sqrt{g}\right)+\Psi(1.2 \sqrt{g})=\left(\frac{\sqrt{2}}{4}-0.2\right) \sqrt{g}+0.6 \sqrt{g}+\frac{1}{1.2} \sqrt{g} \\
& =\left(\frac{\sqrt{2}}{4}+0.4+\frac{5}{6}\right) \sqrt{g}=\frac{15 \sqrt{2}+74}{60} \sqrt{g}>1.5 \sqrt{g}=\max _{t \in I} c(t, P) .
\end{aligned}
$$

This contradicts the fact that $l(S)$ joins the deviating group $T$.

CASE 2: $|T| \geq\left|S_{j}\right|=s_{j}$. Since $\left|l(S)-x_{j-1}\right| \leq 0.1 \sqrt{g}$, we have $l(S) \in S_{j}$ or $l(S) \in S_{j-1}$, so $\sqrt{2 g} \leq\left|S^{l(S)}\right| \leq|T|$. Hence, $c(l(S), T) \geq \Psi(|T|) \geq \Psi\left(S^{l(S)}\right)=c\left(l(S), S^{l(S)}\right)=c(l(S), P)$, so the individual $l(S)$ does not improve from $P$ to $T$. This contradiction proves our proposition.

Proof of Proposition 4.5. Since any homogenous consecutive partition satisfies BI, Lemma A.8 applies, so $P$ is CS if no interval can deviate.

Part (i), $n>1$; "only if". We have already checked (see the proof of Proposition 4.1 (ii), "only if" part), that for $s<\sqrt{g}$ our partition will not be CS.

If $s>(2+\sqrt{2}) \sqrt{g}$, then $T=\left[x_{1}-\frac{\sqrt{g}}{2}, x_{1}+\frac{\sqrt{g}}{2}\right]$, the interval of the size $\sqrt{g}$ centered in one of the peripheral points of $P$ would benefit from deviation. Indeed, among the members of $T$, the 
individual $x_{1}-\frac{\sqrt{g}}{2}$ has the largest cost in $T$ and the smallest cost in $P$, but still

$$
c\left(x_{1}-\frac{\sqrt{g}}{2}, P\right)=\Psi(s)-\frac{\sqrt{g}}{2}>\frac{1+\sqrt{2}}{2} \sqrt{g}+\frac{\sqrt{g}}{2+\sqrt{2}}=1.5 \sqrt{g}=c\left(x_{1}-\frac{\sqrt{g}}{2}\right) .
$$

Part (i), $n>1$; "if". If $s \in[\sqrt{g}, 2 \sqrt{g}]$ then Proposition B.1 (utilized in the proof of Proposition 4.2 above) guarantees that $P$ is CS. Let $s \in(2 \sqrt{g},[2+\sqrt{2}] \sqrt{g}]$, so that $1.5 \leq \Psi(s) \leq 2 \sqrt{g}$, and let $T=[l(S), r(S)]$ be a deviating interval.

Similarly to Lemma A.6, there exist peripheral (relative to jurisdictions in $P$ ) individuals $x_{i}$ and $x_{j}$, such that $\left|l(S)-x_{i}\right|,\left|r(S)-x_{j}\right|<0.6 \sqrt{g}$. Indeed, without loss of generality assume $l(S) \in S_{i}=\left[x_{i-1}, x_{i}\right] \in P, l(S) \geq m\left(S_{i}\right)$. Then: $\left|l(S)-x_{i}\right|=c\left(x_{i}, S_{i}\right)-c\left(l(S), S_{i}\right)=\Psi(s)-$ $c(l(S), P)<2 \sqrt{g}-1.4 \sqrt{g}=0.6 \sqrt{g}$.

Now, since $2 \sqrt{g} \leq s$, we have $i \geq j$.

If $i=j$, then assume $l(S) \in S_{i}$ (if not, then $r(S) \in S_{i+1}$ and we do the same argument for $\left.c\left(r(S), S_{i+1}\right)\right)$. We then obtain the following contradiction with $T$ deviating profitably:

$$
\begin{aligned}
& c\left(l(S), S_{i}\right)=\Psi(s)-\left|x_{i}-p_{i}\right|<\Psi(s)-0.6 \sqrt{g} \leq 1.4 \sqrt{g}=\frac{42}{30} \sqrt{g}, \\
& c(l(S), T) \geq \Psi(|T|) \geq \Psi(1.2 \sqrt{g})=\frac{43}{30} \sqrt{g} .
\end{aligned}
$$

If $i<j$, then we can assume without loss of generality that $a_{1}=\left|l(S)-x_{i}\right| \geq a_{2}=\left|r(S)-x_{j}\right|$.

Using Remark A.1, we again obtain a contradiction with $T$ profitably deviating:

$$
c(l(S), T)=\Psi(|T|)=\Psi\left((j-i) s \pm a_{1} \pm a_{2}\right)>\Psi(s)-\frac{\left| \pm a_{1} \pm a_{2}\right|}{2} \geq \Psi(s)-\left|a_{1}\right|=c(l(S), P) .
$$

This completes the proof of part (i).

Part (ii), $n=1$. Let $P=\{I\}$, and suppose that all members of $T=[l(S), r(S)]$ benefit from the deviation by $T$. Without loss of generality, let $m(T)<m(I)=\frac{1}{2}$. If $r(S) \geq \frac{1}{2}$, then individual $r(S)$ would be worse off at $T$ than at $I$ (indeed, both monetary and transportation cost would increase). Hence, $r(S)<\frac{1}{2}$.

Denote $p=r(S)-l(S)$. We claim that $T^{\prime}=[0, p]$ also can deviate under CS. Indeed, for every individual $t \in T^{\prime}$ we have $c\left(t, T^{\prime}\right)=c(t+l(S), T)<c(t+l(S), I) \leq c(t, I)$. Next, if $T^{\prime}=[0, p]$, where $p<\frac{1}{2}$, can deviate under CS, then (since members of $T^{\prime}$ contribute at least $2 g$ ) $2 g<c\left(t, T^{\prime}\right)<c(t, I) \leq \frac{1}{2}+g$, which implies $g<\frac{1}{2}$. Hence, the partition $\{I\}$ is CS if $g \geq \frac{1}{2}$. 
Further, interval $T^{\prime}=[0, p]$ can deviate if and only if $\Delta\left(t, T^{\prime},\{I\}\right)<0$ for all $t \in T^{\prime}$. But

$$
\Delta\left(t, T^{\prime},\{I\}\right)=c\left(t, T^{\prime}\right)-c(t, I)= \begin{cases}\Psi(p)-\Psi(1), & 0 \leq t \leq \frac{p}{2} \\ \Psi(p)-\Psi(1)+(2 t-p), & \frac{p}{2} \leq t \leq p\end{cases}
$$

is increasing on $[0, p]$, hence, $T=[0, p]$ can deviate if and only if $\Delta(p, T,\{I\})<0$. Now

$$
\Delta(p, T,\{I\})=c\left(p, T^{\prime}\right)-c(p, I)=\left(\frac{p}{2}+\frac{g}{p}\right)-\left(\frac{1}{2}-p+g\right)=-\left(\frac{1}{2}+g\right)+\left(\frac{3}{2} p+\frac{g}{p}\right) .
$$

For given $g$, this function is a convex in $p$, and attains its minimum at $p^{*}=\sqrt{2 g / 3}$. Hence, an interval $[0, p]$ that can profitably deviate exists if and only if $\Delta\left(p^{*},\left[0, p^{*}\right],\{I\}\right)<0$. Finally,

$$
\Delta\left(p^{*},\left[0, p^{*}\right],\{I\}\right)=-\left(\frac{1}{2}+g\right)+\left(\frac{3}{2} p^{*}+\frac{g}{p^{*}}\right)=\sqrt{6 g}-\left(\frac{1}{2}+g\right) .
$$

Thus, $P=\{I\}$ is not CS if and only if $g>\frac{1}{2}$ and $\sqrt{6 g}-g-\frac{1}{2}<0$. That is, $\sqrt{g}<\frac{1}{2+\sqrt{6}}$.

\section{References}

Alesina, A. and E. Spolaore (1997), On the number and size of nations, Quarterly Journal of Economics 112, 1027-1056.

Bogomolnaia, A., Le Breton, M., Savvateev, A. and S. Weber (2005a), The egalitarian sharing rule in provision of public projects, Economics Bulletin 8 (11), 1-5.

Bogomolnaia, A., Le Breton, M., Savvateev, A. and S. Weber (2005b), Stability of jurisdiction structures under the equal share and median rules, CORE Discussion Paper.

Bogomolnaia, A., Le Breton, M., Savvateev, A. and S. Weber (2006), Heterogeneity Gap in Stable Jurisdiction Structures, mimeo.

Cassela, A. (2001), The role of market size in the formation of jurisdictions, Review of Economic Studies 68, 83-108.

Cremer, H., de Kerchove, A.-M. and J. Thisse (1985), An economic theory of public facilities in space, Mathematical Social Sciences 9, 249-262.

Dahm, M., Cechlárová, K., and V. Lasko (2001), Efficiency and stability in a discrete model of country formation, Journal of Global Optimization 20, 239-256. 
Drèze, H. Le Breton, M., and S. Weber (2006) Rawlsian pricing of access to public facilities: a unidimensional illustration, mimeo.

Greenberg, J. and S. Weber (1986), Strong Tiebout equilibrium under restricted preferences domain, Journal of Economic Theory 38, 101-117.

Guesnerie, R. and C. Oddou (1979), On economic games that are not necessarily superadditive, Economics Letters 3, 301-306.

Jéhiel, P. and S. Scotchmer (1997), Free mobility and the optimal number of jurisdictions, Annals d'Economie et Statistiques 45, 219-231.

Jéhiel, P. and S. Scotchmer (2001), Constitutional rules of exclusion in jurisdiction formation, Review of Economic Studies 68, 393-413.

Haeringer, G. (2000), Stable coalition structures with fixed division scheme, in Lecture Notes in Economic and Mathematical Systems, Kirman, A. and J.-B. Zimmermann, eds., Springer Verlag 503.

Haimanko, O., Le Breton, M. and S. Weber (2004), Voluntary formation of communities for provision of public projects, Journal of Economic Theory 115, 1-34.

Haimanko, O., Le Breton, M. and S. Weber (2005), Transfers in a polarized country: bridging the gap between efficiency and stability, Journal of Public Economics, 89, 1277-1303.

Le Breton, M. and S. Weber (2003), The art of making everybody happy: how to prevent a secession, IMF Stuff Papers, 50, 403-435.

Le Breton, M. and S. Weber (2004), Secession-proof cost allocations and stable group structures in models of horizontal differentiation, in Group Formation in Economics: Networks, Clubs and Coalitions, Demange, G. and M. Wooders, eds., Cambridge University Press, 266-285.

Le Breton, M., Weber, S. and J.H. Drèze (2004), The Rawlsian principle and secession-proofness in large heterogeneous societies, CORE Discussion Paper. 